\title{
Synthesis of Novel Purinyl-1'-homocarbanucleosides Based on a Cyclopenta[b]pyrazine System
}

\author{
Carmen Balo, ${ }^{a}$ Carmen López, ${ }^{*}, a$ Olga CAamaño, ${ }^{a}$ Franco Fernández,${ }^{a}$ Xerardo García-Mera, ${ }^{a}$ and \\ José Enrique RodríGUEZ-Borges ${ }^{b}$ \\ ${ }^{a}$ Departamento de Química Orgánica, Facultade de Farmacia, Universidade de Santiago de Compostela; 15782-Santiago \\ de Compostela, Spain: and ${ }^{b}$ CIQ. Departamento de Química da Facultade de Ciencias do Porto; Ruado Campo Alegre, \\ 687-4169007 Porto, Portugal. \\ Received November 29, 2007; accepted February 2, 2008; published online February 14, 2008
}

cis-2,3-Diphenyl-6,7-dihydro-5H-cyclopenta[b]pyrazine-5,7-dimethanol, prepared by Diels-Alder reaction from cyclopentadiene and appropriately protected 2 -imidazolone-followed by dihydroxylation, glycol protection, diamine deprotection, condensation with benzyl, glycol deprotection, oxidative cleavage and reduction-, was used to synthesize ( \pm )-cis-\{[7-(6-chloro-9H-purin-9-yl)methyl]-2,3-diphenyl-6,7-dihydro-5H-cyclopenta[b]pyrazin-5-yl\}methanol, a key intermediate for novel 1'-homocarbanucleosides based on a cyclopenta[b]pyrazine scaffold as shown by its conversion into several 6-substituted purinyl derivatives.

Key words imidazolone; oxidative cleavage; hydroxylation; dihydropyrazine; epimerization; homocarbanucleoside

The success of abacavir (1) as reverse transcriptase inhibitor in human immunodeficiency (HIV) clinical trials, ${ }^{1-4)}$ which led to its approval by FDA for treatment of HIV infection in 1998, has been attributed in large part to the rigidity afforded by its double bond between $\mathrm{C} 2^{\prime}$ and $\mathrm{C} 3^{\prime 5,6)}$ and to its cyclopropylamino group, which increases its lipophilicity and its ability to penetrate into the central nervous system, an important reservoir of the HIV and other viruses. ${ }^{7}$

Inspired on that, our research group has been investigating the biological properties of other carbanucleosides as well as their 1'-homo-counterparts, in which the planar region of the carbocyclic double bond present in abacavir is replaced by a benzene ring ${ }^{8-10)}$ or by an aromatic heterocycle, ${ }^{11-13)}$ seeking to modify the lipophilicity and polar interactions of this region of the molecule while preserving its rigidity. Most of the new compounds showed little if any antiviral activity against HIV, but in a broader antiviral survey some of them in which there is a pyrazol ring fused to the cyclopentene ring such as in $\mathbf{2}$, proved to be highly active against varicellazoster virus and cytomegalovirus at subcytotoxic concentration, ${ }^{12)}$ while an important number of both indan and cyclopenta $[c]$ pyrazole derivatives were found to have considerable cytostatic activity against human T lymphocytes (Molt4/ $\mathrm{C} 8$ and CEM/0 cells). ${ }^{8,9,13)}$

These results pushed us to further explore the field of compounds in which an heterocycle is fused to the cyclopentene moiety of the carbanucleoside. Here we report a synthetic way that leads to 1 '-homonucleoside analogues of the type $\mathrm{A}$, based on a 6,7-dihydro- $5 H$-cyclopenta[b]pyrazine scaffold.
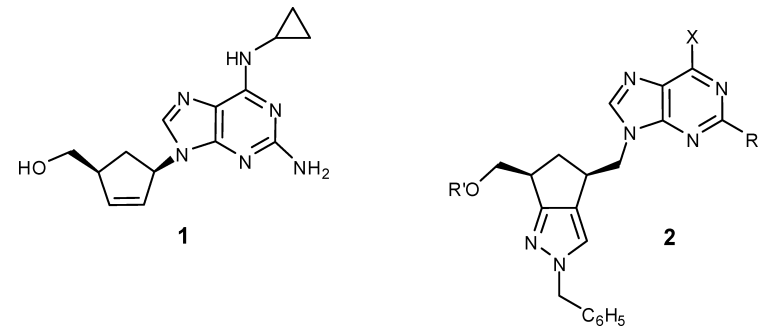

* To whom correspondence should be addressed.

e-mail: qomcls@usc.es

\section{Results and Discussion}

Our work was initially planned on a convergent synthesis (Chart 1) that brings together the preformed main blocs of the final structure through a Mitsunobu coupling, as in some previous analogues. ${ }^{13)}$ Now, while several purine derivatives are commercially available, this strategy compelled us to achieve a properly functionalised cyclopenta[$[b]$ pyrazine derivative, and a dimethanol derivative of the type $\mathrm{B}$ was envisaged as the key synthetic intermediate. B would be available through a sequence based on the retrosynthetic pathway $\mathrm{B} \Rightarrow \mathrm{E}$ depicted in Chart 1 .

Following 1,3-diacetylation of 2-imidazolone, compound 3 (Chart 2) was obtained as per Whitney ${ }^{14)}$ Hydroxylation with $\mathrm{OsO}_{4}$ and methylmorpholine $N$-oxide ${ }^{15}$ in acetone/water afforded a $74 \%$ yield of glycol 4 , which upon treatment with dimethoxypropane gave acetonide $\mathbf{5}$. An attempt to convert 5 into diamine 7 in one step by heating with $\mathrm{KOH}$ in methanol at $155^{\circ} \mathrm{C}$ afforded a complex mixture including only traces of the desired product, while a two-steps process, heating of compound 5 with $\mathrm{KOH}$ in methanol at $65^{\circ} \mathrm{C}$, isolation of the resulting imidazolone $\mathbf{6}$, and heating of $\mathbf{6}$ with $\mathrm{KOH}$ in methanol at $155^{\circ} \mathrm{C}$ in a sealed tube, ${ }^{16)}$ afforded a good yield of a product that could be by identified as 7 by ${ }^{1} \mathrm{H}-\mathrm{NMR}$ spectroscopy. Due to the lability and volatility of this kind of compounds, ${ }^{16)} 7$ was used in the next step without further pu-

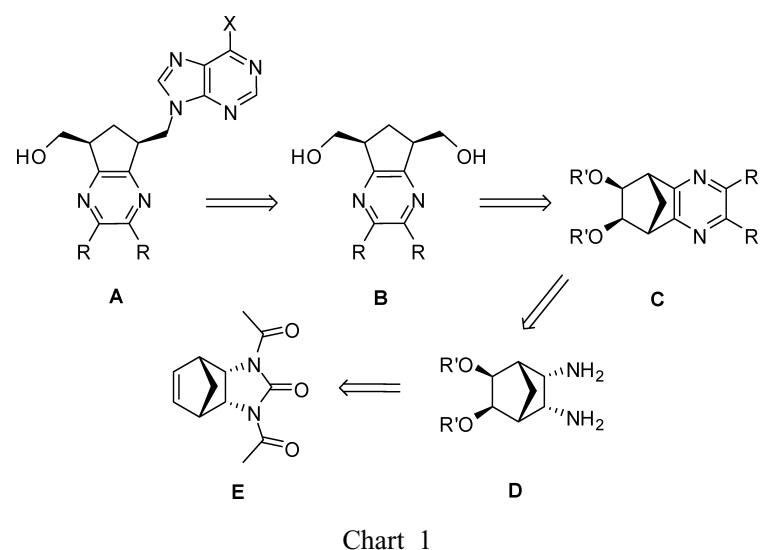

Chart 1 


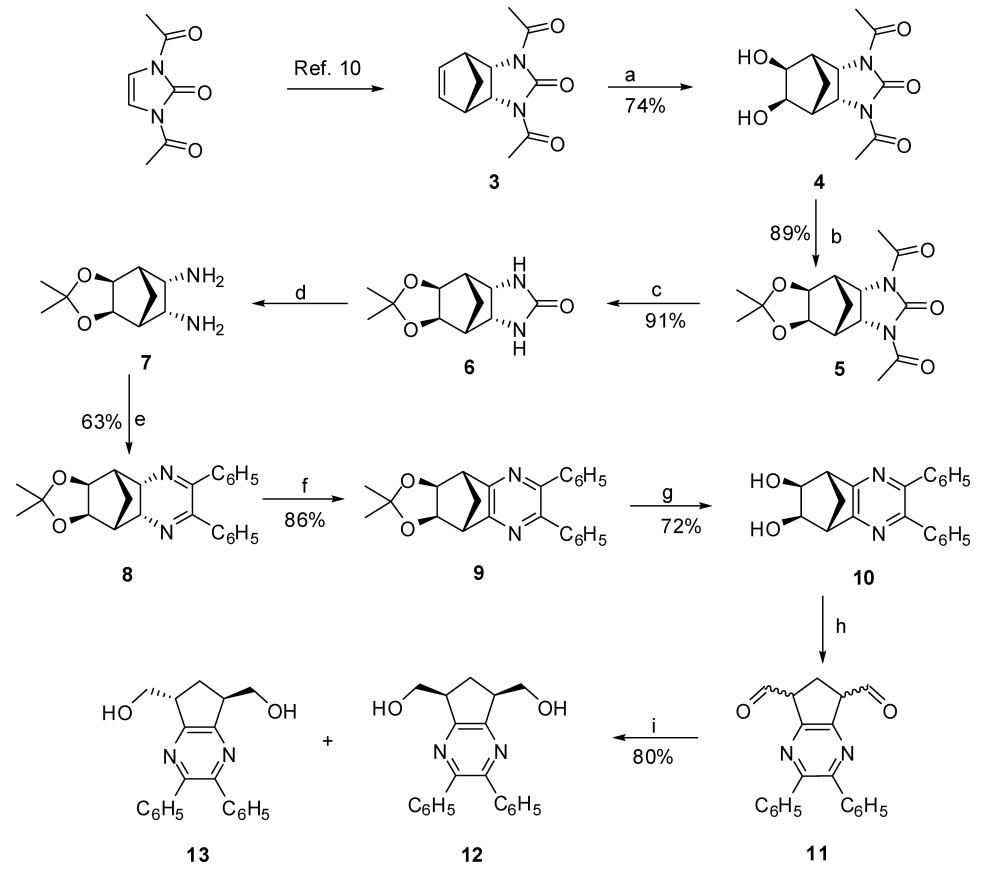

Reaction conditions: a) $\mathrm{OsO}_{4}, \mathrm{NMO}, 40^{\circ} \mathrm{C}$; b) 2,2-dimethoxypropane/ $\mathrm{H}^{+}$, r.t.; c) $\mathrm{KOH} / \mathrm{MeOH}$, reflux. $5 \mathrm{~h}$; d) $\mathrm{KOH} / \mathrm{MeOH}, 155^{\circ} \mathrm{C}, 48 \mathrm{~h}$; e) benzil/THF/ $\mathrm{H}^{+}$, reflux; f) DDQ/toluene, reflux. $2.5 \mathrm{~h} ; \mathrm{g}$ ) $\mathrm{HCl} / \mathrm{EtOH}, 70^{\circ} \mathrm{C}, 10 \mathrm{~h}$; h) $\mathrm{NaIO}_{4} /$ silica gel $\mathrm{CH}_{2} \mathrm{Cl}_{2}$, r.t.; i) $\mathrm{NaBH}_{4} / \mathrm{MeOH}$, r.t.

Chart 2

rification. Thus, crude 7 was condensed with benzil in THF with acetic acid as catalyst, and the resulting dihydropyrazine (8) was aromatized in $86 \%$ yield by refluxing with 4,5 dichloro-3,6-dioxo-1,4-cyclohexadiene-1-2-dicarbonitrile (DDQ) in toluene for $2.5 \mathrm{~h}$. Attempts to achieve mild cleavage of the acetonide moiety of compound 9 (treatment with $60 \% \mathrm{AcOH}$ or $\mathrm{HCO}_{2} \mathrm{H}$, at r.t. or under reflux, or with $\mathrm{HClO}_{4}$ or $\mathrm{BF}_{3} \cdot \mathrm{OEt}_{2}$ at r.t.) were all unsuccessful, the starting material being recovered or intractable mixtures of unidentified products being obtained. Finally diol $\mathbf{1 0}$ was prepared in good yield by moderate heating of $\mathbf{9}$ in $\mathrm{EtOH}$ in presence of $\mathrm{HCl}$.

Oxidative cleavage of $\mathbf{1 0}$ with sodium periodate on silica gel ${ }^{17)}$ then gave dialdehyde 11 (as identified by IR spectroscopy), and reduction of crude $\mathbf{1 1}$ with $\mathrm{NaBH}_{4}$ in methanol finally afforded an approximately $2: 1$ mixture of the desired cis-diol 12 and its diastereomer 13; partial epimerization is presumed to have occurred during these last two steps.

Diastereomers 12 and $\mathbf{1 3}$ were clearly distinguished and cis/trans relative configurations initially assigned to them on the basis of their ${ }^{1} \mathrm{H}-\mathrm{NMR}$ spectra: each of the chemically unequivalent $\mathrm{C} 6$ protons of cis-diol $\mathbf{1 2}$ gives rise to a doublet of triplets, at $\delta 1.61(J=9.3,13.1 \mathrm{~Hz})$ and $\delta 2.51(J=8.3$, $13.1 \mathrm{~Hz}$ ), while the two chemically equivalent $\mathrm{C} 6$ protons of trans-diol $\mathbf{1 3}$ give rise to a single triplet at $\delta 2.18$ $(J=7.3 \mathrm{~Hz})$. Compound $\mathbf{1 3}$ was then conclusively identified by X-ray crystallography (Fig. 1). ${ }^{18)}$

Several attempts to perform Mitsunobu processes with 6chloropurine on $\mathbf{1 2}$ or monoprotected derivatives of it were fruitless and we then turned to more classical nuecleophilic displacements. Esterification of $\mathbf{1 2}$ with acetic anhydride and pyridine afforded a mixture including the starting compound, its diacetate, and the monoacetate $\mathbf{1 4}$, which was converted into mesylate 15 in $69 \%$ yield by treatment with mesyl chlo-

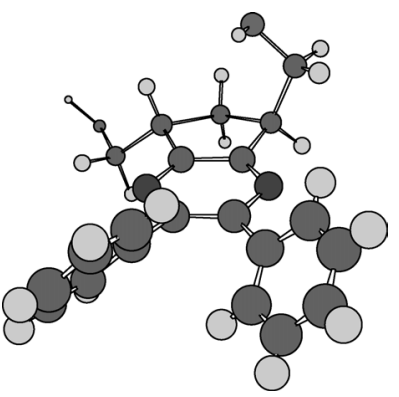

Fig. 1. ORTEP Projection of the Molecular Structure of Compound $\mathbf{1 3}$

ride, triethylamine and 4-(dimethylamino)pyridine (DMAP) at $0^{\circ} \mathrm{C}$ (Chart 2). Coupling 15 with 6-chloropurine by reaction in DMF in the presence of $\mathrm{NaH}$ and 18 -crown-6 ether ${ }^{11)}$ finally afforded nucleoside analogue $\mathbf{1 6}$ in 37\% yield; an alkene, thought to be $\mathbf{1 7}$ (two singlets at 5.36 and $6.17 \mathrm{ppm}$ in the ${ }^{1} \mathrm{H}-\mathrm{NMR}$ spectra) and formed from 15, presumably through a dehydromesyloxylation promoted by the basic medium,${ }^{11)}$ was also detected as the main by-product (in an estimated $25 \%$ yield).

Suitability of chloropurine $\mathbf{1 6}$ to serve as an appropriate key intermediate for the preparation of nucleoside analogues of this new class was shown through its smooth conversion into methoxypurine analogue $\mathbf{1 8}$ by treatment with methanol and aqueous $\mathrm{HCl}$ at room temperature (r.t.), and into amino derivatives 19 and 20 , by reaction with liquid ammonia and cyclopropylamine, respectively.

In conclusion, this paper describes a convenient synthetic procedure for the preparation of novel purinyl-1'-homocarbanucleoside derivatives 16, 18, 19 and 20 , as examples of an interesting new template in which the double bond of the cyclopentenyl nucleosides is embedded in a pyrazine ring, thus opening a route to a wide variety of other purinyl derivatives 

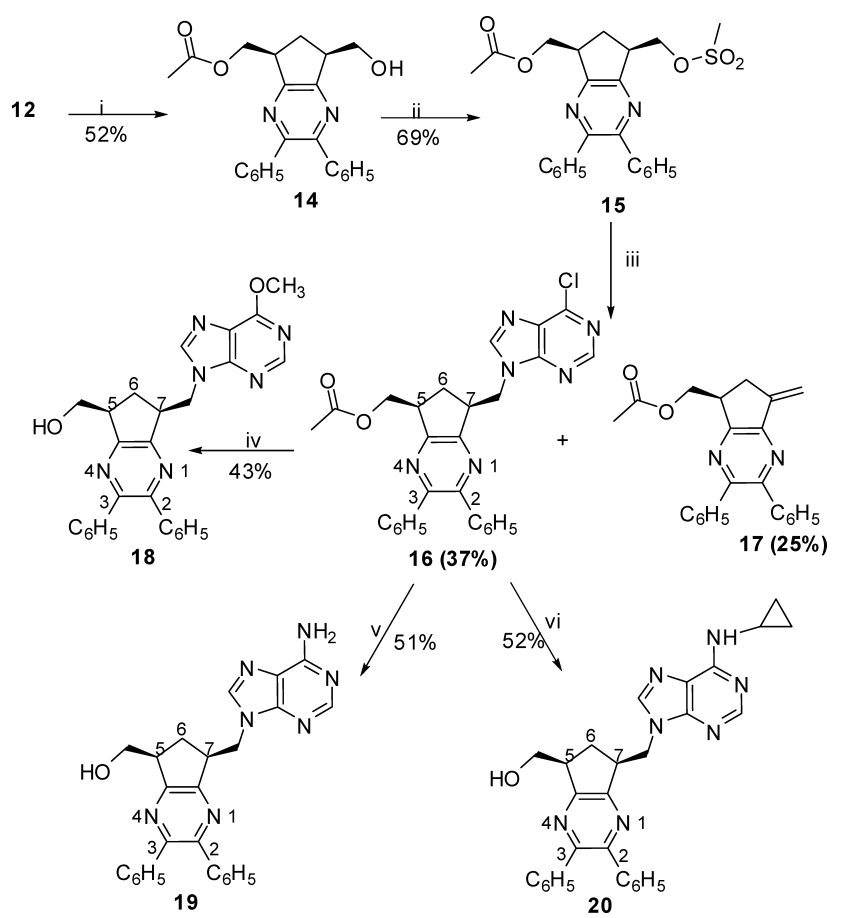

Reaction conditions: i) $\mathrm{Ac}_{2} \mathrm{O} / \mathrm{Pyr}$, r.t.; ii) $\mathrm{MeSO}_{2} \mathrm{Cl}, \mathrm{NEt}_{3} / \mathrm{DMAP}$;ii) 6-chloropurine $\mathrm{NaH}, 18$-crow-6 ether; $\mathrm{HCl}, \mathrm{MeOH}$, r.t.; iv) $\mathrm{MeOH} / \mathrm{HCl}$, r.t.; v) $\mathrm{MeOH} / \mathrm{NH}_{3}, 75^{\circ} \mathrm{C}$; vi) cyclopropylamine/EtOH, reflux.

Chart 3

with similar cyclopenta[$[b]$ pyrazine scaffolds.

\section{Experimental}

All chemical used were of reagent grade and were obtained from Aldrich Chemical Co. and used without further purification. Melting points were measured in a Reichert Kofler Thermopan and are uncorrected. Infrared spectra were recorded on a Perkin-Elmer 1640 FT-IR spectrophotometer. ${ }^{1} \mathrm{H}-$ and ${ }^{13} \mathrm{C}-\mathrm{NMR}$ spectra were recorded in a Bruker AMX-300 spectrometer at 300 and $75 \mathrm{MHz}$, respectively, using TMS as internal standard (chemical shifts in $\delta$ values, $J$ in $\mathrm{Hz}$ ). Microanalyses were performed in a LECO CHNS-932 Elemental Analyser at the University of Santiago Microanalysis Service. Analyses indicated by the symbols of elements were within $\pm 0.4 \%$ of the theoretical values. Flash chromatography was performed on silica gel (Merck 60, 230-240 mesh) and analytical TCL on pre-coated silica gel plates (Merck $60, \mathrm{~F}_{254}, 0.25 \mathrm{~mm}$ ). MPLC separations were carried out using a Biotage Flash 4Di system with a Biotage column (Si-40B, silica gel). XRay diffraction data were collected in an Enraf-Nonius CAD4 automatic diffractometer using the programme CAD4-EXPRESS.

$(3 \mathrm{a} R, 4 R, 5 R, 6 S, 7 S, 7 \mathrm{a} S)$-1,3-Diacetyl-5,6-dihydroxyperhydro-4,7methanobenzo-imidazol-2-one (4) A solution of $3(4.0 \mathrm{~g}, 17 \mathrm{mmol})$ in (4:1 acetone/water) $(35 \mathrm{ml})$ was heated to $40^{\circ} \mathrm{C}$ and methylmorpholine $\mathrm{N}$ oxide $(2.2 \mathrm{~g}, 18.9 \mathrm{mmol})$ was added, followed after $5 \mathrm{~min}$ at the same temperature by a $4 \%(\mathrm{w} / \mathrm{w})$ solution of $\mathrm{OsO}_{4}$ in water $(0.5 \mathrm{ml})$, which immediately caused the mixture to turn brown. After $90 \mathrm{~min}$ stirring at $40^{\circ} \mathrm{C}$ the reaction mixture was filtered through celite, concentrated, and treated with saturated $\mathrm{NH}_{4} \mathrm{Cl}$ solution. This mixture was extracted with EtOAc, the organic phase was washed with brine and dried over $\mathrm{Na}_{2} \mathrm{SO}_{4}$, the solvent was removed under reduced pressure, and purification of the residue by chromatography on silica gel with $1: 1$ hexane/EtOAc as eluent afforded $\mathbf{4}$ as a white solid $(3.55 \mathrm{~g}$, yield $74 \%)$. A sample was recrystallized from toluene for analysis, $\mathrm{mp} 178-179^{\circ} \mathrm{C}$. IR $(\mathrm{KBr}) \mathrm{cm}^{-1}: 3479,2957,1755,1683,1274$, 1239. ${ }^{1} \mathrm{H}-\mathrm{NMR}\left(\mathrm{CDCl}_{3}\right) \delta: 1.39(1 \mathrm{H}, \mathrm{d}, J=11.7 \mathrm{~Hz}, 8-\underline{\mathrm{HH}}), 2.04(1 \mathrm{H}, \mathrm{d}$, $J=11.7 \mathrm{~Hz}, 8-\mathrm{H} \underline{\mathrm{H}}), 2.53\left(6 \mathrm{H}, \mathrm{s}, 2 \times \mathrm{CH}_{3}\right), 2.69\left(2 \mathrm{H}, \mathrm{s}, 2 \times \mathrm{OH}, \mathrm{D}_{2} \mathrm{O}\right.$ exch. $)$, $2.83(2 \mathrm{H}, \mathrm{s}, 4 \mathrm{H}+7 \mathrm{H}), 3.76(2 \mathrm{H}, \mathrm{s}, 5 \mathrm{H}+6 \mathrm{H}), 4.27(2 \mathrm{H}, \mathrm{s}, 3 \mathrm{aH}+7 \mathrm{aH}) .{ }^{13} \mathrm{C}-$ $\operatorname{NMR}\left(\mathrm{CDCl}_{3}\right) \delta: 24.80\left(\mathrm{CH}_{3}\right), 31.16\left(\mathrm{CH}_{2}\right), 46.76(\mathrm{CH}), 54.16(\mathrm{CH}), 68.99$ (CH), 152.55 (CO), 172.00 (CO). Anal. Calcd for $\mathrm{C}_{12} \mathrm{H}_{16} \mathrm{~N}_{2} \mathrm{O}_{5}$ (268.27): C, 53.73 ; H, 6.01; N, 10.44. Found: C, 53.70, H, 5.99, N, 10.43 .

(3aR,4R,4a R,7aS,8S,8aS)-5,7-Diacetyl-2,2-dimethylperhydro-4,8methano[1,3]dioxolo[4,5- $f$ ]benzoimidazol-6-one (5) A solution of diol 4 $(3.18 \mathrm{~g}, 11.9 \mathrm{mmol})$ in 2,2-dimethoxypropane $(8 \mathrm{ml})$ containing a small amount of $p$-toluenesulphonic acid was stirred at r.t. until reaction was judged to be complete on the basis of TLC monitoring ( $12 \mathrm{~h})$. The reaction mixture was then washed with saturated $\mathrm{NaHCO}_{3}$ solution and extracted with $\mathrm{CH}_{2} \mathrm{Cl}_{2}$, and the organic phase was dried with $\mathrm{Na}_{2} \mathrm{SO}_{4}$ and concentrated to dryness under reduced pressure, affording $\mathbf{5}$ as a white solid (3.23 g, yield $89 \%$ ) from which a sample was taken and recrystallized from toluene for analysis, mp $176-177^{\circ} \mathrm{C}$. IR ( $\left.\mathrm{KBr}\right) \mathrm{cm}^{-1}$ : 2991, 2944, 1746, 1691, 1368, $1322,1277,1238,1056 .{ }^{1} \mathrm{H}-\mathrm{NMR}\left(\mathrm{CDCl}_{3}\right) \delta: 1.24\left(3 \mathrm{H}, \mathrm{s}, \mathrm{CH}_{3}\right), 1.24-1.29$ $(1 \mathrm{H}, \mathrm{m}, 9-\underline{\mathrm{HH}}), 1.42\left(3 \mathrm{H}, \mathrm{s}, \mathrm{CH}_{3}\right), 1.87(1 \mathrm{H}, \mathrm{d}, J=11.6 \mathrm{~Hz}, 9-\mathrm{HH}), 2.52(6 \mathrm{H}$, s, $\left.2 \times \mathrm{CH}_{3}\right), 2.92-2.94(2 \mathrm{H}, \mathrm{m}, 4-\mathrm{H}+8-\mathrm{H}), 3.98(2 \mathrm{H}, \mathrm{d}, J=1.4 \mathrm{~Hz}, 3 \mathrm{a}-$ $\mathrm{H}+8 \mathrm{a}-\mathrm{H}), 4.29(2 \mathrm{H}, \mathrm{t}, J=2.3 \mathrm{~Hz}, 4 \mathrm{a}-\mathrm{H}+7 \mathrm{a}-\mathrm{H}) .{ }^{13} \mathrm{C}-\mathrm{NMR}\left(\mathrm{CDCl}_{3}\right) \delta: 24.33$ $\left(\mathrm{CH}_{3}\right), 24.73\left(\mathrm{CH}_{3}\right), 25.73\left(\mathrm{CH}_{3}\right), 30.84\left(\mathrm{CH}_{2}\right), 43.55(\mathrm{CH}), 53.60(\mathrm{CH})$, $76.81(\mathrm{CH}), 109.45(\mathrm{C}), 152.41(\mathrm{CO}), 171.12$ (CO). Anal. Calcd for $\mathrm{C}_{15} \mathrm{H}_{20} \mathrm{~N}_{2} \mathrm{O}_{5}$ (308.33): C, 58.43; H, 6.49; N, 9.09. Found: C, 58.67, H, 6.69, $\mathrm{N}, 10.13$.

$(3 \mathrm{a} R, 4 R, 4 \mathrm{a} R, 7 \mathrm{a} S, 8 S, 8 \mathrm{a} S)$-2,2-Dimethylperhydro-4,8-methano[1,3]dioxolo[4,5- $f$ benzoimidazol-6-one (6) A suspension of $5(3.05 \mathrm{~g}$, $9.9 \mathrm{mmol})$, methanol $(12 \mathrm{ml})$ and $50 \% \mathrm{KOH}(4.5 \mathrm{ml})$ was refluxed for $5 \mathrm{~h}$ with TLC monitoring, and the solids were then filtered out, washed repeatedly with water, and dried to constant mass $(1.67 \mathrm{~g})$ in a vacuum desiccator. The filtrate was neutralized and extracted with $\mathrm{CHCl}_{3}$, and the $\mathrm{CHCl}_{3}$ phase was dried and concentrated to dryness under reduced pressure, yielding a solid $(0.32 \mathrm{~g})$ with the same chromatographic $R f$ as the filtration residue. Total yield, $91 \%$. A sample was taken and recrystallized from toluene for analysis, $\mathrm{mp} 310-312^{\circ} \mathrm{C}$. IR $(\mathrm{KBr}) \mathrm{cm}^{-1}: 3358,3236,2977,1693,1379$, $1249,1213,1039,860 .{ }^{1} \mathrm{H}-\mathrm{NMR}$ (DMSO- $\left.d_{6}\right) \delta: 1.05(1 \mathrm{H}, \mathrm{d}, J=10.7 \mathrm{~Hz}, 9-$ $\mathrm{HH}), 1.20\left(3 \mathrm{H}, \mathrm{s}, \mathrm{CH}_{3}\right), 1.33\left(3 \mathrm{H}, \mathrm{s}, \mathrm{CH}_{3}\right), 1.53(1 \mathrm{H}, \mathrm{d}, J=10.7 \mathrm{~Hz}, 9-\mathrm{HH})$, $2.24(2 \mathrm{H}, \mathrm{s}, 4-\mathrm{H}+8-\mathrm{H}), 3.84(2 \mathrm{H}, \mathrm{s}, 3 \mathrm{a}-\mathrm{H}+8 \mathrm{a}-\mathrm{H}), 4.29(2 \mathrm{H}, \mathrm{s}, 4 \mathrm{a}-\mathrm{H}+7 \mathrm{a}-\mathrm{H})$, $6.37\left(2 \mathrm{H}, 2 \times \mathrm{NH}\right.$, exch. $\left.\mathrm{D}_{2} \mathrm{O}\right) .{ }^{13} \mathrm{C}-\mathrm{NMR}$ (DMSO- $\left.d_{6}\right) \delta: 24.13\left(\mathrm{CH}_{3}\right), 25.78$ $\left(\mathrm{CH}_{3}\right), 30.13\left(\mathrm{CH}_{2}\right), 44.06(\mathrm{CH}), 53.68(\mathrm{CH}), 77.13(\mathrm{CH}), 107.85(\mathrm{C})$, 162.48 (CO). Anal. Calcd for $\mathrm{C}_{11} \mathrm{H}_{16} \mathrm{~N}_{2} \mathrm{O}_{3}$ (224.25): C, 58.91; H, 7.19; N, 12.49. Found: C, 59.08; H, 7.32, N, 12.61.

$(3 \mathrm{a} R, 4 R, 5 R, 6 S, 7 S, 7 \mathrm{a} S)$-2,2-Dimethylperhydro-4,7-methano-1,3-benzodioxole-5,6-diamine (7) A suspension of $6(1.9 \mathrm{~g}, 8.5 \mathrm{mmol})$ in methanol $(8.6 \mathrm{ml})$ and $50 \% \mathrm{KOH}(21 \mathrm{ml})$ was heated at $155^{\circ} \mathrm{C}$ in a sealed tube for $48 \mathrm{~h}$, allowed to cool to r.t., concentrated, and extracted with $\mathrm{CHCl}_{3}$. The $\mathrm{CHCl}_{3}$ phase was dried over $\mathrm{Na}_{2} \mathrm{SO}_{4}$, and removal of the solvent under reduced pressure left a yellow solid $(1.63 \mathrm{~g})$ that was used in the next step without further purification. ${ }^{1} \mathrm{H}-\mathrm{NMR}$ (DMSO- $\left.d_{6}\right) \delta: 1.03-1.08(1 \mathrm{H}, \mathrm{m}, 8-$ $\underline{\mathrm{HH}}), 1.20-1.58\left(11 \mathrm{H}, \mathrm{m}, 2 \times \mathrm{CH}_{3}+8-\mathrm{H} \underline{\mathrm{H}}+2 \times \mathrm{NH}_{2}\right.$, exch. four with $\left.\mathrm{D}_{2} \mathrm{O}\right)$, $2.64(2 \mathrm{H}, \mathrm{s}, 4-\mathrm{H}+7-\mathrm{H}), 3.34(2 \mathrm{H}, \mathrm{s}, 3 \mathrm{a}-\mathrm{H}+7 \mathrm{a}-\mathrm{H}), 4.32(2 \mathrm{H}, \mathrm{s}, 5-\mathrm{H}+6-\mathrm{H})$.

$(3 \mathrm{a} R, 4 R, 4 \mathrm{a} R, 8 \mathrm{a} S, 9 S, 9 \mathrm{a} S)$-2,2-Dimethyl-6,7-diphenyl-3a,4,4a,8a,9,9ahexahydro-4,9-methano[1,3]dioxolo[4,5-g]quinoxaline (8) A solution of benzil $(1.72 \mathrm{~g}, 8.2 \mathrm{mmol})$ in THF $(6 \mathrm{ml})$ was added to a solution of crude 7 $(1.63 \mathrm{~g}, 8.2 \mathrm{mmol})$ in the same solvent $(20 \mathrm{ml})$, a catalytic amount of acetic acid was added, and the mixture was refluxed until reaction was deemed complete on the basis of TLC monitoring (4h). Concentration to dryness left a yellow solid that was purified by chromatography on silica gel with $(2: 1)$ hexane/EtOAc as eluent followed by recrystallization from ethanol $(1.92 \mathrm{~g}$, yield $63 \%$ ), mp $166-167^{\circ} \mathrm{C}$. IR (KBr) cm ${ }^{-1}: 2984,2912,1570,1461$, 1206, 1057. ${ }^{1} \mathrm{H}-\mathrm{NMR}\left(\mathrm{CDCl}_{3}\right) \delta: 1.16-1.20\left(4 \mathrm{H}, \mathrm{m}, \mathrm{CH}_{3}+10-\underline{\mathrm{HH}}\right), 1.39$ $\left(3 \mathrm{H}, \mathrm{s}, \mathrm{CH}_{3}\right), 1.73(1 \mathrm{H}, \mathrm{d}, J=10.7 \mathrm{~Hz}, 10-\mathrm{H}-1), 2.98(2 \mathrm{H}, \mathrm{m}, 4-\mathrm{H}+9-\mathrm{H})$, $4.19-4.21(2 \mathrm{H}, \mathrm{m}, 3 \mathrm{a}-\mathrm{H}+9 \mathrm{a}-\mathrm{H}), 4.22(2 \mathrm{H}, \mathrm{d}, J=1.2 \mathrm{~Hz}, 4 \mathrm{a}-\mathrm{H}+8 \mathrm{a}-\mathrm{H})$, 7.03-7.21 (10H, m, arom). ${ }^{13} \mathrm{C}-\mathrm{NMR}\left(\mathrm{CDCl}_{3}\right) \delta: 24.29\left(\mathrm{CH}_{3}\right), 25.80$ $\left(\mathrm{CH}_{3}\right), 27.18\left(\mathrm{CH}_{2}\right), 47.05(\mathrm{CH}), 54.69(\mathrm{CH}), 78.04(\mathrm{CH}), 108.90(\mathrm{C})$, $128.24(\mathrm{CH}), 128.44(\mathrm{CH}), 129.39(\mathrm{CH}), 139.37(\mathrm{C}), 156.76(\mathrm{C})$. Anal. Calcd for $\mathrm{C}_{24} \mathrm{H}_{24} \mathrm{~N}_{2} \mathrm{O}_{2}$ (372.46): C, 77.39; H, 6.49; N, 7.52. Found: C, 77.18; $\mathrm{H}, 6.75, \mathrm{~N}, 7.33$.

(3a $R, 4 R, 9 S, 9 \mathrm{a} S)$-2,2-Dimethyl-6,7-diphenyl-3a,4,9,9a-tetrahydro-4,9methano[1,3]-dioxolo[4,5-g]quinoxaline (9) A solution of DDQ $(1.09 \mathrm{~g}$, $4.8 \mathrm{mmol})$ in toluene $(20 \mathrm{ml})$ was added to a solution of $8(1.79 \mathrm{~g}, 4.8 \mathrm{mmol})$ in the same solvent $(28 \mathrm{ml})$, and the mixture was refluxed under argon for $2.5 \mathrm{~h}$, vacuum filtered, and concentrated to dryness, leaving a reddish solid that was recrystallized from EtOH/EtOAc $(1.53 \mathrm{~g}$, yield $86 \%), \mathrm{mp} 237$ $239^{\circ} \mathrm{C}$. IR (KBr) cm ${ }^{-1}: 2984,2907,1454,1360,960 .{ }^{1} \mathrm{H}-\mathrm{NMR}\left(\mathrm{CDCl}_{3}\right) \delta$ : $1.30\left(3 \mathrm{H}, \mathrm{s}, \mathrm{CH}_{3}\right), 1.51\left(3 \mathrm{H}, \mathrm{s}, \mathrm{CH}_{3}\right), 2.19(1 \mathrm{H}, \mathrm{dd}, J=1.4,10.1 \mathrm{~Hz}, 10-\underline{\mathrm{HH}})$, $2.48(1 \mathrm{H}, \mathrm{d}, J=10.1 \mathrm{~Hz}, 10-\mathrm{HH}), 3.50(2 \mathrm{H}, \mathrm{s}, 4-\mathrm{H}+9-\mathrm{H}), 4.47(2 \mathrm{H}, \mathrm{d}$, $J=1.2 \mathrm{~Hz}, 3 \mathrm{a}-\mathrm{H}+9 \mathrm{a}-\mathrm{H}), 7.16-7.32\left(10 \mathrm{H}, \mathrm{m}\right.$, arom.). ${ }^{13} \mathrm{C}-\mathrm{NMR}\left(\mathrm{CDCl}_{3}\right) \delta$ : $24.75\left(\mathrm{CH}_{3}\right), 26.15\left(\mathrm{CH}_{3}\right), 41.61\left(\mathrm{CH}_{2}\right), 48.77(\mathrm{CH}), 80.86(\mathrm{CH}), 114.20$ (C), $128.55(\mathrm{CH}), 128.68(\mathrm{CH}), 130.15(\mathrm{CH}), 139.39(\mathrm{C}), 150.50(\mathrm{C})$, 158.89 (C). Anal. Calcd for $\mathrm{C}_{24} \mathrm{H}_{22} \mathrm{~N}_{2} \mathrm{O}_{2}$ (370.45): C, 77.81; H, 5.99; N, 7.56. Found: C, 77.53, H, 6.11, N, 7.38.

$(5 R, 6 R, 7 S, 8 S)$-2,3-Diphenyl-5,6,7,8-tetrahydro-5,8-methanoquinoxaline-6,7-diol (10) $12 \mathrm{~N} \mathrm{HCl}(1.5 \mathrm{ml})$ was added to a suspension of 9 
$(1.31 \mathrm{~g}, 3.54 \mathrm{mmol})$ in ethanol $(60 \mathrm{ml})$ at $0{ }^{\circ} \mathrm{C}$, and the mixture was heated at $70{ }^{\circ} \mathrm{C}$ until TLC showed no starting compound $(10 \mathrm{~h})$. Following removal of the solvent under reduced pressure, the residue was taken into methanol and passed through an ion-exchange column (Amberlite IRA $400 \mathrm{OH}^{-}$), and the methanolic eluate was concentrated to dryness. Column chromatography of the residue on silica gel with $3: 1$ hexane/EtOAc as eluent afforded a solid $(0.84 \mathrm{~g}$, yield $72 \%)$, a sample of which was recrystallized from hexane/EtOAc for analysis, mp $185-187^{\circ} \mathrm{C}$. IR (KBr) cm ${ }^{-1}$ : 3384, 1449, 1364, 1222, 1144, 949. ${ }^{1} \mathrm{H}-\mathrm{NMR}\left(\mathrm{CDCl}_{3}\right) \delta: 2.15(1 \mathrm{H}, \mathrm{d}, J=10.1 \mathrm{~Hz}, 9-$ $\underline{\mathrm{HH}}), 2.44(1 \mathrm{H}, \mathrm{d}, J=10.1 \mathrm{~Hz}, 9-\mathrm{H} \underline{\mathrm{H}}), 3.42(2 \mathrm{H}, \mathrm{s}, 5-\mathrm{H}+8-\mathrm{H}), 3.97(2 \mathrm{H}, \mathrm{s}, 6-$ $\mathrm{H}+7-\mathrm{H}), 4.37\left(2 \mathrm{H}\right.$, br s, $2 \times \mathrm{OH}$, exch. $\left.\mathrm{D}_{2} \mathrm{O}\right), 7.26-7.19(10 \mathrm{H}, \mathrm{m}$, arom. $)$. ${ }^{13} \mathrm{C}-\mathrm{NMR}\left(\mathrm{CDCl}_{3}\right) \delta: 42.22\left(\mathrm{CH}_{2}\right), 51.59(\mathrm{CH}), 70.41(\mathrm{CH}), 128.44(\mathrm{CH})$, $128.68(\mathrm{CH}), 130.13(\mathrm{CH}), 138.42(\mathrm{C}), 146.78(\mathrm{C}), 157.77(\mathrm{C})$. Anal. Calcd for $\mathrm{C}_{21} \mathrm{H}_{18} \mathrm{~N}_{2} \mathrm{O}_{2}$ (330.38): C, 76.34; H, 5.49; N, 8.48. Found: C, 76.00, H, $5.68, \mathrm{~N}, 8.81$.

2,3-Diphenyl-6,7-dihydro-5 $H$-cyclopenta[b]pyrazine-5,7-dicarbaldehyde (mixture of cis and ( \pm )-trans) (11) A $0.65 \mathrm{M}$ aqueous solution of $\mathrm{NaIO}_{4}(6.06 \mathrm{ml})$ was added to a suspension of silica gel $(5.9 \mathrm{~g})$ in $\mathrm{CH}_{2} \mathrm{Cl}_{2}$ $(50 \mathrm{ml})$, and the mixture was shaken vigorously by hand and, following the formation of a flaky precipitate, added to a solution of $\mathbf{1 0}(1.0 \mathrm{~g}, 3.03 \mathrm{mmol})$ in $\mathrm{CH}_{2} \mathrm{Cl}_{2}(6.0 \mathrm{ml})$. This mixture was stirred at room temperature until TLC showed no starting compound $(15 \mathrm{~min})$, and was then filtered through $\mathrm{Na}_{2} \mathrm{SO}_{4}$ and concentrated under reduced pressure, affording a vivid orange solid that was identified as compound $\mathbf{1 1}$ (on the basis of the strong carbonyl band at $1723 \mathrm{~cm}^{-1}$ in its IR spectrum) and was used without further purification in the next step.

cis-2,3-Diphenyl-6,7-dihydro-5 $H$-cyclopenta[b]pyrazine-5,7-dimethanol (12) and ( \pm )-trans-2,3-Diphenyl-6,7-dihydro-5H-cyclopenta[b]pyrazine-5,7-dimethanol (13) $\quad \mathrm{NaBH}_{4}(56.74 \mathrm{mg}, 1.5 \mathrm{mmol})$ was added to a solution of $\mathbf{1 0}(90 \mathrm{mg}, 0.27 \mathrm{mmol})$ in $\mathrm{MeOH}(15 \mathrm{ml})$ and the mixture was left stirring (colour change). When TLC showed no remaining starting compound $(30 \mathrm{~min})$, water $(10 \mathrm{ml})$ was added and stirring was continued for $15 \mathrm{~min}$, after which the methanol was removed under reduced pressure and the remaining aqueous solution was extracted with EtOAc. The organic phase was dried with $\mathrm{Na}_{2} \mathrm{SO}_{4}$ and concentrated to dryness, leaving a solid that following chromatography on a silica gel column with $(1: 2)$ hexane/EtOAc as eluent afforded a solid foam ( $81 \mathrm{mg}$, yield $80 \%)$ with two components. Further chromatography on an MPLC column with $(98: 2)$ $\mathrm{CH}_{2} \mathrm{Cl}_{2} / \mathrm{MeOH}$ as eluent afforded the $12(52 \mathrm{mg})$ followed by the 13 (29 mg).

Compound 12: $\mathrm{mp} 126-128^{\circ} \mathrm{C}$ (from EtOAc/Et $\mathrm{O}$ ). IR $(\mathrm{KBr}) \mathrm{cm}^{-1}$ : $3411,2899,1594,1449,1386,1238,1059,950 .{ }^{1} \mathrm{H}-\mathrm{NMR}\left(\mathrm{CDCl}_{3}\right) \delta: 1.61$ $(1 \mathrm{H}, \mathrm{dt}, J=9.3,13.1 \mathrm{~Hz}, 6-\underline{\mathrm{H}}), 2.51(1 \mathrm{H}, \mathrm{dt}, J=8.3,13.1 \mathrm{~Hz}, 6-\mathrm{H} \underline{\mathrm{H}})$, $3.51-3.71\left(4 \mathrm{H}, \mathrm{m}, 5-\mathrm{H}+7-\mathrm{H}+2 \times \mathrm{OH}\right.$ (two of them $\mathrm{D}_{2} \mathrm{O}$ exch.), $3.92(2 \mathrm{H}$, dd, $J=7.9, \quad 10.8 \mathrm{~Hz}, 2 \times \mathrm{C} \underline{\mathrm{H} H O H}), 4.05 \quad(2 \mathrm{H}, \quad \mathrm{dd}, J=4.9, \quad 10.8 \mathrm{~Hz}$, $2 \times \mathrm{CH} \underline{\mathrm{HOH}}), 7.24-7.41\left(10 \mathrm{H}, \mathrm{m}\right.$, arom.). ${ }^{13} \mathrm{C}-\mathrm{NMR}\left(\mathrm{CDCl}_{3}\right) \delta: 28.09$ $\left(\mathrm{CH}_{2}\right), 44.10(\mathrm{CH}), 66.07\left(\mathrm{CH}_{2}\right), 128.63(\mathrm{CH}), 128.94(\mathrm{CH}), 130.19(\mathrm{CH})$, 139.04 (C), 151.56 (C), 158.63 (C). Anal. Calcd for $\mathrm{C}_{21} \mathrm{H}_{20} \mathrm{~N}_{2} \mathrm{O}_{2}(332.40)$ : C, 75.88; H, 6.06; N, 8.43. Found: C, 76.08, H, 6.18, N, 8.69.

Compound 13: mp 207-209 ${ }^{\circ} \mathrm{C}$ (from EtOAc/Et ${ }_{2} \mathrm{O}$ ). IR $(\mathrm{KBr}) \mathrm{cm}^{-1}$ : 3380, 3225, 2921, 2864, 1459, 1387, 1141, 772. ${ }^{1} \mathrm{H}-\mathrm{NMR}\left(\mathrm{CDCl}_{3}\right) \delta: 1.65$ $(1 \mathrm{H}$, br s, OH, D $\mathrm{O}$ exch. $), 2.18(2 \mathrm{H}, \mathrm{t}, J=7.3 \mathrm{~Hz}, 6-\underline{\mathrm{HH}}), 3.08(1 \mathrm{H}$, br s, $\mathrm{OH}, \mathrm{D}_{2} \mathrm{O}$ exch.) $3.51-3.61(2 \mathrm{H}, \mathrm{m}, 5-\mathrm{H}+7-\mathrm{H}), 3.91(2 \mathrm{H}, \mathrm{dd}, J=7.8$, $10.5 \mathrm{~Hz}, 2 \times \mathrm{CHHOH}), 4.02(2 \mathrm{H}, \mathrm{dd}, J=5.9,10.5 \mathrm{~Hz}, 2 \times \mathrm{CH} \underline{\mathrm{HOH}}), 7.13$ $7.52\left(10 \mathrm{H}, \mathrm{m}\right.$, arom.). ${ }^{13} \mathrm{C}-\mathrm{NMR}\left(\mathrm{CDCl}_{3}\right) \delta: 27.38\left(\mathrm{CH}_{2}\right), 43.89(\mathrm{CH}), 65.84$ $\left(\mathrm{CH}_{2}\right), 128.12(\mathrm{CH}), 128.40(\mathrm{CH}), 128.68(\mathrm{CH}), 138.53(\mathrm{C}), 151.15(\mathrm{C})$, $157.62(\mathrm{C})$. Single crystals suitable for X-ray diffractometry were obtained by dissolving a sample of $\mathbf{1 3}$ in the least possible quantity of cold EtOAc in an open vial that was then placed in a larger container with a little hexane at the bottom; the container was closed, and after a few days in a cool, dark place free from vibrations some single crystals were formed, one of which was analysed by X-ray diffractometry.

( \pm )-cis-[7-(Hydroxymethyl)-2,3-diphenyl-6, 7-dihydro-5Hcyclopenta[b]pyrazin-5-yl]methyl Acetate (14) Acetic anhydride $(180 \mathrm{mg}, 1.8 \mathrm{mmol})$ was added to a solution of $12(60 \mathrm{mg}, 0.18 \mathrm{mmol})$ in pyridine $(2 \mathrm{ml})$, the mixture was stirred under argon at r.t. for $12 \mathrm{~h}$, water was added, and following extraction with AcOEt the organic phase yielded a residue from which chromatography on silica gel with hexane/EOtAc $(3: 1)$ as eluent separated the diester $(12 \mathrm{mg})$, the desired monoester 14 (as oil) $(53 \mathrm{mg}$, yield $52 \%)$, and starting diol $12(10 \mathrm{mg})$. IR (film) $\mathrm{cm}^{-1}: 3360$, $1738,1443,1247,1038,782 .{ }^{1} \mathrm{H}-\mathrm{NMR}\left(\mathrm{CDCl}_{3}\right) \delta: 1.76(1 \mathrm{H}, \mathrm{dt}, J=8.8$, $13.2 \mathrm{~Hz}, 6-\underline{\mathrm{HH}}), 2.07\left(3 \mathrm{H}, \mathrm{s}, \mathrm{CH}_{3}\right), 2.62(1 \mathrm{H}, \mathrm{dt}, J=8.6,13.3 \mathrm{~Hz}, 6-\mathrm{H} \underline{\mathrm{H}})$, $3.53(1 \mathrm{H}, \mathrm{dq}, J=5.3,8.6 \mathrm{~Hz}, 7-\mathrm{H}), 3.63-3.72(2 \mathrm{H}, \mathrm{m}, \mathrm{CHHOH}+5-\mathrm{H}), 3.93$ $(1 \mathrm{H}, \mathrm{dd}, J=8.1,10.6 \mathrm{~Hz}, \mathrm{CH}-\mathrm{HH}), 4.03\left(1 \mathrm{H}\right.$, br s, OH, exch. $\left.\mathrm{D}_{2} \mathrm{O}\right), 4.39$
(1H, dd, $J=7.1,11.1 \mathrm{~Hz}$, C $\underline{H} H O A c), 4.72(1 \mathrm{H}, \mathrm{dd}, J=4.6,11.1 \mathrm{~Hz}$,

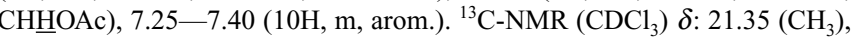
$29.39\left(\mathrm{CH}_{2}\right), 42.14(\mathrm{CH}), 43.63(\mathrm{CH}), 66.19\left(\mathrm{CH}_{2}\right), 66.32\left(\mathrm{CH}_{2}\right), 128.60$ $(\mathrm{CH}), 128.87(\mathrm{CH}), 128.92(\mathrm{CH}), 130.22(\mathrm{CH}), 139.08(\mathrm{C}), 139.26(\mathrm{C})$ 155.85 (C), 158.41 (C), 171.53 (CO). Anal. Calcd for $\mathrm{C}_{23} \mathrm{H}_{22} \mathrm{~N}_{2} \mathrm{O}_{3}$ (374.44): C, 73.78; H, 5.94; N, 7.48. Found: C, 73.69; H, 6.05; N, 7.22.

(士)-cis-\{[7-(Methanesulfonyloxymethyl)-2,3-diphenyl-6,7-dihydro-5Hcyclopenta[b]pyrazin-5-yl]\}methyl Acetate (15) To a solution of 14 $(400 \mathrm{mg}, 1.07 \mathrm{mmol})$ in dry chloroform $(5 \mathrm{ml})$ in an ice bath were added $\mathrm{Et}_{3} \mathrm{~N}(0.5 \mathrm{ml})$ and a catalytic amount of DMAP. Following dropwise addition of mesyl chloride $(0.25 \mathrm{ml}, 3.2 \mathrm{mmol})$, the mixture was stirred at r.t. for $2 \mathrm{~h}$, refluxed for $12 \mathrm{~h}$ with TLC monitoring, poured over ice-water, and left stirring for $1 \mathrm{~h}$, after which the organic phase was drawn off, washed with $1 \mathrm{~N}$ $\mathrm{NaOH}$ and brine, dried with $\mathrm{Na}_{2} \mathrm{SO}_{4}$ and concentrated under reduced pressure, affording compound $\mathbf{1 5}$ as an oil that was purified by column chromatography with $(2: 1)$ hexane/AcOEt as eluent $(330 \mathrm{mg}$, yield $69 \%)$. IR (film) $\mathrm{cm}^{-1}: 3450,3025,2989,1735,1456,1285,1250,1045,977,879$, 755. ${ }^{1} \mathrm{H}-\mathrm{NMR}\left(\mathrm{CDCl}_{3}\right) \delta: 1.94-2.04(1 \mathrm{H}, \mathrm{m}, 6-\underline{\mathrm{HH}}), 2.08\left(3 \mathrm{H}, \mathrm{s}, \mathrm{CH}_{3} \mathrm{CO}\right)$, $2.77(1 \mathrm{H}, \mathrm{dt}, J=8.8,13.5 \mathrm{~Hz}, 6-\mathrm{HH}), 2.99\left(3 \mathrm{H}, \mathrm{s}, \mathrm{CH}_{3} \mathrm{SO}_{3}\right), 3.65-3.73(2 \mathrm{H}$, m, 5-H+7-H), $4.40(1 \mathrm{H}, \mathrm{dd}, J=6.8,9.8 \mathrm{~Hz}, \mathrm{C} \underline{\mathrm{H}}), 4.62(1 \mathrm{H}, \mathrm{dd}, J=6.8$, $10.0 \mathrm{~Hz}, \mathrm{CHH}), 4.71-4.81(2 \mathrm{H}, \mathrm{m}, 2 \times \mathrm{CHH}), 7.26-7.54(10 \mathrm{H}, \mathrm{m}$, arom. $)$ ${ }^{13} \mathrm{C}-\mathrm{NMR}\left(\mathrm{CDCl}_{3}\right) \delta: 21.35\left(\mathrm{CH}_{3}\right), 28.35\left(\mathrm{CH}_{2}\right), 38.01\left(\mathrm{CH}_{3} \mathrm{SO}_{3}\right), 40.21$ $(\mathrm{CH}), 42.57(\mathrm{CH}), 67.90\left(\mathrm{CH}_{2}\right), 68.85\left(\mathrm{CH}_{2}\right), 128.01(\mathrm{CH}), 128.77(\mathrm{CH})$, 129.33 (CH), 138.26 (C), 138.97 (C), 155.60 (C), 158.32 (C), 171.50 (CO) Anal. Calcd for $\mathrm{C}_{24} \mathrm{H}_{24} \mathrm{~N}_{2} \mathrm{O}_{5} \mathrm{~S}$ (452.52): C, 63.70; H, 5.35; N, 6.18; S, 7.08. Found: C, 63.98; H, 5.55; N, 6.34; S, 6.89.

( \pm )-cis-\{[7-(6-Chloro-9H-purin-9-yl)methyl]-2,3-diphenyl-6,7-dihydro-5 $\boldsymbol{H}$-cyclopenta[b]pyrazin-5-yl]\}methyl Acetate (16) A solution of 6chloropurine $(148 \mathrm{mg}, 0.96 \mathrm{mmol}), \mathrm{NaH}(24 \mathrm{mg}, 0.96 \mathrm{mmol})$ and 18 -crown6 ether $(170 \mathrm{mg}, 0.66 \mathrm{mmol})$ in DMF $(8 \mathrm{ml})$ was heated to $55^{\circ} \mathrm{C}$, a solution of mesylate $15(300 \mathrm{mg}, 0.66 \mathrm{mmol})$ in the same solvent $(4 \mathrm{ml})$ was added, and heating was continued for $20 \mathrm{~h}$. The solvent was removed under reduced pressure, and chromatography of the residue on silica gel with (1:1) hexane/EtOAc as eluent finally afforded compound $\mathbf{1 6}$ as a white solid $(180 \mathrm{mg}$, yield $37 \%)$, mp $196-198^{\circ} \mathrm{C}$. IR $(\mathrm{KBr}) \mathrm{cm}^{-1}: 3323,2990,1739$, 1594, 1336, 1044, 768. ${ }^{1} \mathrm{H}-\mathrm{NMR}\left(\mathrm{CDCl}_{3}\right) \delta: 1.86(1 \mathrm{H}, \mathrm{dt}, J=8.8,13.2 \mathrm{~Hz}$, $6-\underline{\mathrm{HH}}), 1.99\left(3 \mathrm{H}, \mathrm{s}, \mathrm{CH}_{3}\right), 2.72(1 \mathrm{H}, \mathrm{dt}, J=8.4,13.2 \mathrm{~Hz}, 6-\mathrm{H} \underline{\mathrm{H}}), 3.60-3.67$ $(1 \mathrm{H}, \mathrm{m}, 5-\mathrm{H}), 3.85-3.93(1 \mathrm{H}, \mathrm{m}, 7-\mathrm{H}), 4.36(1 \mathrm{H}, \mathrm{dd}, J=6.6,11.0 \mathrm{~Hz}, 5-$ $\mathrm{C} \underline{\mathrm{HH}}), 4.64(1 \mathrm{H}, \mathrm{dd}, J=4.2,11.1 \mathrm{~Hz}, 5-\mathrm{CH} \underline{\mathrm{H}}), 4.87$ and $4.78(2 \mathrm{H}, \mathrm{AB}$ part of an $\mathrm{ABX}$ system, $\left.J_{\mathrm{AX}}=6.0, J_{\mathrm{BX}}=6.2, J_{\mathrm{AB}}=14.1 \mathrm{~Hz}, 7-\mathrm{CH}_{2}\right), 7.26-7.50$ $(10 \mathrm{H}, \mathrm{m}$, arom. $), 8.29$ and $8.72\left(2 \mathrm{H}, 2 \mathrm{~s}, 2^{\prime}-\mathrm{H}+8^{\prime}-\mathrm{H}\right) .{ }^{13} \mathrm{C}-\mathrm{NMR}\left(\mathrm{CDCl}_{3}\right) \delta$ : $20.89\left(\mathrm{CH}_{3}\right), 30.86\left(\mathrm{CH}_{2}\right), 41.17(\mathrm{CH}), 42.25(\mathrm{CH}), 46.31\left(\mathrm{CH}_{2}\right), 65.15$ $\left(\mathrm{CH}_{2}\right), 128.12(\mathrm{CH}), 128.18(\mathrm{CH}), 128.55(\mathrm{CH}), 128.89(\mathrm{C}), 129.50(\mathrm{CH})$, $129.66(\mathrm{CH}), 129.77(\mathrm{C}), 138.30(\mathrm{C}), 138.39(\mathrm{C}), 146.01(\mathrm{CH}), 151.32(\mathrm{C})$, $151.81(\mathrm{CH}), 152.02(\mathrm{C}), 152.22$ (C), 154.36 (C), 154.93 (C), 170.73 (CO). Anal. Calcd for $\mathrm{C}_{26} \mathrm{H}_{21} \mathrm{ClN}_{6} \mathrm{O}$ (464.94): C, 66.82; H, 4.54; N, 17.45. Found: $\mathrm{C}, 66.59, \mathrm{H}, 4.51, \mathrm{~N}, 17.92$

( \pm )-cis-\{[7-(6-Methoxy-9H-purin-9-yl)methyl]-2,3-diphenyl-6,7-dihydro-5H-cyclopenta $[b \mid$ pyrazin-5-yl $]$ methanol (18) A solution of 16 $(305 \mathrm{mg}, 0.59 \mathrm{mmol}), \mathrm{MeOH}(4 \mathrm{ml})$ and $\mathrm{HCl} 1 \mathrm{~N}(4 \mathrm{ml})$ was stirred at r.t. for $14 \mathrm{~h}$, and after removal of the solvent the resulting residue was dissolved in EtOAc $(20 \mathrm{ml})$. This solution was washed with brine $(20 \mathrm{ml})$ and the solvent removed under reduced pressure, leaving a oil $(260 \mathrm{mg})$ that after chromatography on silica gel with $\mathrm{CH}_{2} \mathrm{Cl}_{2}-\mathrm{MeOH}(92: 8)$ as eluent afforded 18 as a oil (120 mg; 43\% yield). IR (film) $\mathrm{cm}^{-1}: 3400,2989,2869,1602,1486$, 1414, 1285, 1045, 977, 879, 755. ${ }^{1} \mathrm{H}-\mathrm{NMR}\left(\mathrm{CDCl}_{3}\right) \delta: 1.71(1 \mathrm{H}, \mathrm{dt}$ $J=13.3 \mathrm{~Hz}, 6-\underline{\mathrm{HH}}), 2.48(1 \mathrm{H}, \mathrm{dt}, J=13.3 \mathrm{~Hz}, 6-\mathrm{H} \underline{\mathrm{H}}), 3.42\left(3 \mathrm{H}, \mathrm{s}, \mathrm{OCH}_{3}\right)$, $3.55-3.62(1 \mathrm{H}, \mathrm{m}, 5-\mathrm{H}), 3.78-3.84\left(2 \mathrm{H}, \mathrm{m}, 5-\mathrm{CH}_{2}\right), 3.98-4.04(1 \mathrm{H}, \mathrm{m}$, 7-H), 4.13 (1H, br s, OH, D 2 exch), 4.55-4.61 (part A of an ABX system $J_{\mathrm{AX}}=5.7, J_{\mathrm{AB}}=14.3 \mathrm{~Hz}, \mathrm{C} \underline{\mathrm{H} H N}$ ) $4.75-4.86$ (part B of an $\mathrm{ABX}$ system $\left.J_{\mathrm{BX}}=7.7, J_{\mathrm{BA}}=14.3 \mathrm{~Hz}, \mathrm{CH} \underline{\mathrm{HN}}\right), 7.19-7.34$ (10H, m, arom.), 7.88 and 8.01 $\left(2 \mathrm{H}, 2 \mathrm{~s}, 2^{\prime}-\mathrm{H}+8^{\prime}-\mathrm{H}\right) .{ }^{13} \mathrm{C}-\mathrm{NMR}\left(\mathrm{CDCl}_{3}\right) \delta: 32.8\left(\mathrm{CH}_{2}\right), 35.2(\mathrm{CH}), 44.2$ $(\mathrm{CH}), 45.8\left(\mathrm{CH}_{3}\right), 46.3\left(\mathrm{CH}_{2}\right), 62.1\left(\mathrm{CH}_{2}\right), 121.1(\mathrm{C}), 127.8(\mathrm{CH}), 128.7$ $(\mathrm{CH}), 128.9(\mathrm{CH}), 129.8(\mathrm{C}), 136.3(\mathrm{CH}), 136.8(\mathrm{C}), 142.0(\mathrm{CH}), 146.5(\mathrm{C})$, 151.4 (C), 152.3 (CH), 158.4 (C), 160.2 (C), 162.3 (C). Anal. Calcd for $\mathrm{C}_{27} \mathrm{H}_{24} \mathrm{~N}_{6} \mathrm{O}_{2}$ (464.52): C, 69.81; H, 5.21; N, 18.09. Found: C, 69.98, H, 5.52, N, 17.86 .

(士)-cis-\{[7-(6-Amino-9H-purin-9-yl)methyl]-2,3-diphenyl-6,7-dihydro-5H-cyclopenta $[b]$ pyrazin-5-yl] $\}$ methanol (19) A solution of 16 $(150 \mathrm{mg}, 0.32 \mathrm{mmol})$ in $\mathrm{MeOH}(4 \mathrm{ml})$ and liquid $\mathrm{NH}_{3}(3 \mathrm{ml})$ was heated in a bomb at $75^{\circ} \mathrm{C}$ for $60 \mathrm{~h}$. Once the reaction mixture had cooled to r.t., the solvent was evapored under reduced pressure and $\mathbf{1 9}$ was isolated as a white solid $(0.07 \mathrm{~g}, 51 \%$ yield $), \mathrm{mp} 224-226\left(\mathrm{Et}_{2} \mathrm{O}\right)$. IR (film) $\mathrm{cm}^{-1}: 3353,1653$, $1556,1418,1319,1238,1074,798,776 .{ }^{1} \mathrm{H}-\mathrm{RMN}\left(\mathrm{CDCl}_{3}\right) \delta: 1.73(1 \mathrm{H}, \mathrm{dt}$, 
$J=8.5,13.1 \mathrm{~Hz}, 6-\underline{\mathrm{HH}}), 2.34(1 \mathrm{H}, \mathrm{dt}, J=8.3,13.3 \mathrm{~Hz}, 6-\mathrm{HH}), 3.54-3.60$ $(1 \mathrm{H}, \mathrm{m}, 5-\mathrm{H}), 3.70-3.79\left(2 \mathrm{H}, \mathrm{m}, 5-\mathrm{CH}_{2}\right), 3.88\left(1 \mathrm{H}, \mathrm{brs}, \mathrm{OH}, \mathrm{D}_{2} \mathrm{O}\right.$ exch. $)$, $4.03-4.12(1 \mathrm{H}, \mathrm{m}, 7-\mathrm{H}), 4.34-4.50$ (part A of an $\mathrm{ABX}$ system $J_{\mathrm{AX}}=5.2$, $J_{\mathrm{AB}}=13.3 \mathrm{~Hz}, \mathrm{C} \underline{\mathrm{HHN}}$ ) $4.55-4.68$ (part B of an $\mathrm{ABX}$ system $J_{\mathrm{BX}}=7.5$, $\left.J_{\mathrm{BA}}=13.3 \mathrm{~Hz}, \mathrm{CH} \underline{\mathrm{HN}}\right), 6.76\left(2 \mathrm{H}, \mathrm{NH}_{2}, \mathrm{D}_{2} \mathrm{O}\right.$ exch). $7.11-7.44(10 \mathrm{H}, \mathrm{m}$, arom.), 7.80 and $7.89\left(2 \mathrm{H}, 2 \mathrm{~s}, 2^{\prime}-\mathrm{H}+8^{\prime}-\mathrm{H}\right) .{ }^{13} \mathrm{C}-\mathrm{NMR}\left(\mathrm{CDCl}_{3}\right) \delta: 31.6$ $\left(\mathrm{CH}_{2}\right), 34.2(\mathrm{CH}), 44.5(\mathrm{CH}), 47.3\left(\mathrm{CH}_{2}\right), 61.2\left(\mathrm{CH}_{2}\right), 120.1(\mathrm{C}), 128.0$ $(\mathrm{CH}), 128.9(\mathrm{CH}), 129.6(\mathrm{C}), 135.4(\mathrm{CH}), 135.7(\mathrm{C}), 141.2(\mathrm{CH}), 145.5(\mathrm{C})$, $150.4(\mathrm{C}), 151.8(\mathrm{CH}), 158.4(\mathrm{C}), 158.2(\mathrm{C}), 160.3(\mathrm{C})$. Anal. Calcd for $\mathrm{C}_{26} \mathrm{H}_{23} \mathrm{~N}_{7} \mathrm{O}$ (449.51). C, 69.47; H, 5.16; N, 21.81.Found: C, 69.32; H, 5.23; $\mathrm{N}, 22.00$.

( \pm )-cis-\{[7-(6-Cyclopropylamino-9H-purin-9-yl)methyl]-2,3-diphenyl6,7-dihydro-5H-cyclopenta[b]pyrazin-5-yl]\}methanol (20) A solution of $16(150 \mathrm{mg}, 0.31 \mathrm{mmol})$ and cyclopropylamine $(127 \mathrm{mg}, 2.22 \mathrm{mmol})$ in EtOH $(10 \mathrm{ml})$ was refuxed for $22 \mathrm{~h}$. Removal of the solvent under reduced pressure left a brown oil from which, following chromatography on silica gel with $\mathrm{CH}_{2} \mathrm{Cl}_{2}-\mathrm{MeOH}(95: 5)$ as eluent, $20(78 \mathrm{mg}, 52 \%)$ was isolated as a ligh oil. IR (film) $\mathrm{cm}^{-1}: 3410,2360,1740,1635,1476,1377,1298,1074$, $768,676 .{ }^{1} \mathrm{H}-\mathrm{NMR}\left(\mathrm{CDCl}_{3}\right) \delta: 0.54-0.58(\mathrm{~m}, 2 \mathrm{H}$, cyclopropyl $), 0.80-0.93$ (m, 2H, cyclopropyl), $1.73(1 \mathrm{H}, \mathrm{dt}, J=8.5,13.1 \mathrm{~Hz}, 6-\underline{\mathrm{H}}), 2.34(1 \mathrm{H}, \mathrm{dt}$, $J=7.3,13.1 \mathrm{~Hz}, 6-\mathrm{H} \underline{\mathrm{H}}), 2.93-2.98\left(\mathrm{~m}, 1 \mathrm{H}, 1_{\text {cyclopropy }} \mathrm{H}\right), 3.54-3.60(1 \mathrm{H}, \mathrm{m}$, $5-\mathrm{H}), 3.79-3.97\left(2 \mathrm{H}, \mathrm{m}, 5-\mathrm{CH}_{2}\right), 4.11\left(1 \mathrm{H}\right.$, br s, OH, $\mathrm{D}_{2} \mathrm{O}$ exch. $), 4.18-$ $4.22(1 \mathrm{H}, \mathrm{m}, 7-\mathrm{H}), 4.35-4.51$ (part $\mathrm{A}$ of an $\mathrm{ABX}$ system $J_{\mathrm{AX}}=5.7$, $J_{\mathrm{AB}}=13.3 \mathrm{~Hz}, \mathrm{C} \underline{H} H \mathrm{~N}$ ), $4.55-4.68$ (part $\mathrm{B}$ of an $\mathrm{ABX}, J_{\mathrm{BX}}=7.5$, $\left.J_{\mathrm{BA}}=13.3 \mathrm{~Hz}, \mathrm{CH} \underline{\mathrm{H}}\right), 7.11-7.44(10 \mathrm{H}, \mathrm{m}$, arom. $), 7.80$ and $7.89(2 \mathrm{H}, 2 \mathrm{~s}$, $\left.2^{\prime}-\mathrm{H}=8^{\prime}-\mathrm{H}\right) .{ }^{13} \mathrm{C}-\mathrm{NMR}\left(\mathrm{CDCl}_{3}\right) \delta: 7.2\left(2 \times \mathrm{CH}_{3}\right), 29.6(\mathrm{CH}), 31.6\left(\mathrm{CH}_{2}\right)$, $34.2(\mathrm{CH}), 44.5(\mathrm{CH}), 47.3\left(\mathrm{CH}_{2}\right), 61.2\left(\mathrm{CH}_{2}\right), 120.1(\mathrm{C}), 128.0(\mathrm{CH}), 128.9$ $(\mathrm{CH}), 129.6(\mathrm{CH}), 135.4(\mathrm{CH}), 135.7(\mathrm{C}), 141.2(\mathrm{CH}), 145.5(\mathrm{C}), 150.4$ $(\mathrm{CH}), 151.8(\mathrm{C}), 158.4(\mathrm{C}), 158.2(\mathrm{C}), 160.3(\mathrm{C})$. Anal. Calcd for $\mathrm{C}_{29} \mathrm{H}_{27} \mathrm{~N}_{7} \mathrm{O}$ (489.58). C, 71.15; H, 5.56; N, 20.03. Found: C, 71.3; H, 5.76; N, 19.88.

Acknowledgements Authors thank Xunta de Galicia for financial support under project PGIDIT02BTF20305PR.

\section{References and Notes}

1) Craul A., Lesson P. A., Castañer J., Drugs Future, 23, 1155-1167 (1998).

2) Dando T. M., Scott L. J., Drugs, 65, 285-302 (2005).

3) Faletto M. B., Miller W. H., Garvey E. P., St. Clair M. H., Daluge S.
M., Good S. S., Antimicrob. Agents Chemother, 41, 1099-1107 (1997).

4) Foster R. H., Faulds D., Drugs, 55, 729-736 (1998).

5) Krayevsky A. A., Watanabe K. A., "Modified Nucleosides as AntiAIDS Drugs: Current Status and Perspectives," Bioinform, Moscow, 1993.

6) Lea A. P., Faulds D., Drugs, 51, 846-852 (1996).

7) Thomas S., McDonall J. E., Cheah V., Bye A., Segal M. B., The Entry of Abacavir into the Guinea Pig Brain: Comparison with Other Reverse Transcriptase Inhibitors; Presented at the 12th World AIDS Conference, Geneva, 1998.

8) Fernández F., García-Mera X., Morales M., Rodríguez-Borges J. E., De Clercq E., Synthesis, 2002, 1084-1091 (2002).

9) Yao S. W., López V. H., Fernández F., García-Mera X., Morales M., Rodríguez-Borges J. E., Cordeiro M. N., Bioorg. Med. Chem., 11, 4999-5006 (2003).

10) Fernández F., García-Mera X., Morales M., Vilariño L., Caamaño O., De Clercq E., Tetrahedron, 60, 9245-9253 (2004).

11) Caamaño O., Gómez G., Fernández F., García M. D., García-Mera X., De Clercq E., Synthesis, 2004, 2855-2861 (2004).

12) García M. D., Caamaño O., Fernández F., López C., De Clercq E., Synthesis, 2005, 925-932 (2005).

13) García M. D., Caamaño O., Fernández F., García-Mera X., Pérez-Castro I., Synthesis, 2006, 3967-3972 (2006).

14) Whitney R. A., Tetrahedron Lett., 22, 2063-2066 (1981).

15) Kobayashi J. E., Kobayashi S., Molecules, 5, 1062-1067 (2000).

16) Eissenstat M. A., Weaver III J. D., J. Org. Chem., 58, 3387-3390 (1993).

17) Zhong Y. L., Shing T. K. M., J. Org. Chem., 62, 2622-2624 (1997).

18) Crystallographic data (excluding structure factors) for the structures 13 in this paper have been deposited with the Cambridge Crystallographic Data Centre as supplementary publication, no. CCDC 242738. Empirical formula: $\mathrm{C}_{21} \mathrm{H}_{20} \mathrm{~N}_{2} \mathrm{O}_{2}$ formula weight: 332.39 ; crystal size: $0.88 \times 0.08 \times 0.04$; crystal system: monoclinic; unit cell dimensions: $a=14.6253(13) \AA, \quad b=6.2834(5) \AA, \quad c=18.6333(7) \AA ; \quad \alpha=90^{\circ}, \quad \alpha=$ $92.811(8)^{\circ}, \gamma=90^{\circ} ; V=1710.3(2) \AA^{3}$; space group: $P_{2}(1) / n ; D_{\text {calc }}=$ $1.291 \mathrm{Mg} / \mathrm{m}^{3} ; F(000)=704 ; \mathrm{R} 1=0.0569$, wR2 $=0.1624$. Diffractometer: Smart-1000 BRUKER. 